\title{
Images of the month 1: Schnitzler syndrome: an acquired autoinflammatory syndrome
}

\author{
Authors: Evangelia Zampeli, ${ }^{A}$ Leonidas Marinos ${ }^{B}$ and Stamatis ] Karakatsanis ${ }^{C}$
}
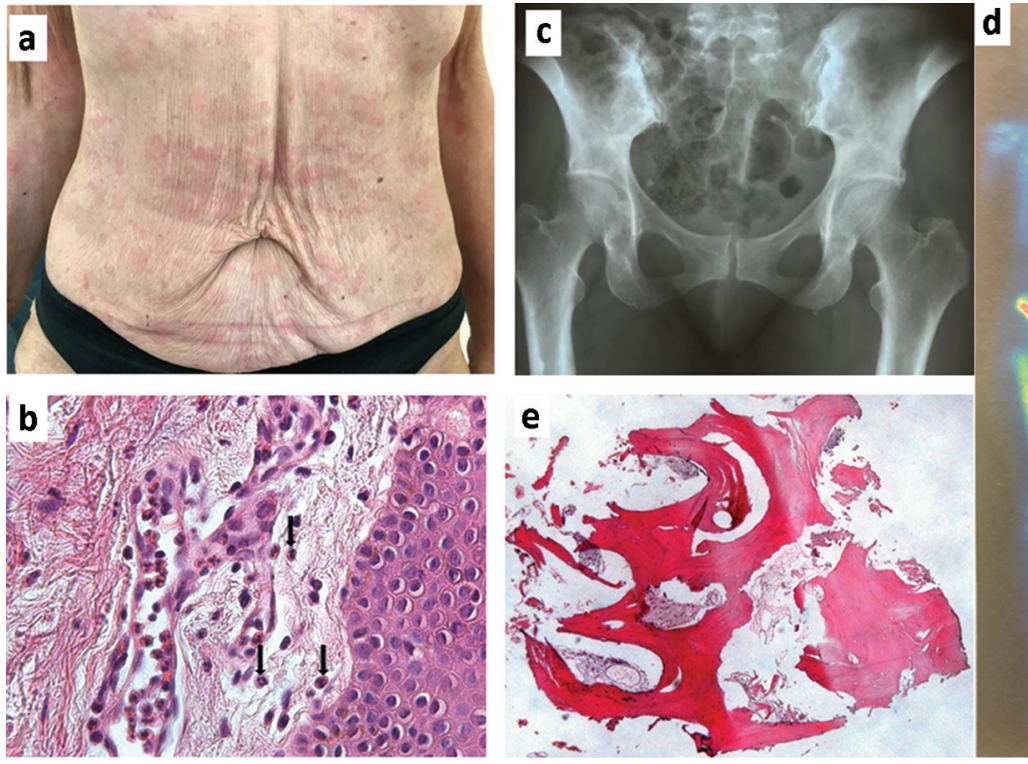

Fig 1. a) A maculopapular urticarial rash on the patient's trunk and arms. b) Skin biopsy (haematoxylin and eosin stain, $100 \times$ magnification) oedema of the dermis, vascular dilatation, presence of scattered polymorphs (neutrophils and eosinophils) and a slight perivascular T-cell (CD3+) lymphocytic infiltrate, findings indicative of urticarial neutrophilic dermatosis. c) Pelvic X-ray showed juxta-articular sclerosis of the ilium, adjacent to the sacroiliac joints symmetrically. d) Bone scintigraphy revealed increased radioactive tracer concentration over the painful bony sites (the pelvic bones and the upper part of the right femur). e) Computed tomography-guided bone biopsy from the right ilium showed increased bony thickness overall / osteosclerosis (haematoxylin and eosin stain, $40 \times$ magnification).
KEYWORDS: Autoinflammatory diseases, periodic fever syndromes, Schnitzler syndrome, neutrophilic dermatosis, monoclonal gammopathy

\section{DOI: 10.7861/clinmed.2019-0466}

\section{Case presentation}

A 55-year-old woman with a 2-year long history of monoclonal gammopathy of unknown significance and a recent diagnosis of 'sacroiliitis' for which she received anti-tumour necrosis factor treatment and was evaluated for high-grade fever and generalised bone pain.

Physical examination revealed a maculopapular urticarial rash on the trunk (Fig 1a) and tenderness over the sacroiliac

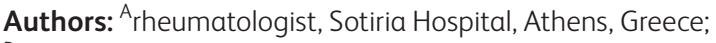
${ }^{B}$ haematopathologist, Evangelismos General Hospital, Athens, Greece; Chaematologist, Sotiria Hospital, Athens, Greece joints, pelvic bones and right femur. Laboratory testing showed mildly elevated white blood cells $\left(12.9 \times 10^{9} / \mathrm{L}\right)$ and acute-phase reactants (C-reactive protein $17.4 \mathrm{mg} / \mathrm{L}$ (normal range $<5 \mathrm{mg} / \mathrm{L}$ ) and erythrocyte sedimentation rate $64 \mathrm{~mm} / \mathrm{h}$ ) and a monoclonal immunoglobulin (IgM) kappa band. Blood cultures, bone-marrow biopsy and computed tomography (CT) of the thorax and abdomen were unrevealing. Skin biopsy showed mild perivascular and interstitial neutrophilic infiltrates in the dermis without vasculitis (Fig 1b), indicative of urticarial neutrophilic dermatosis. Pelvic X-ray showed osteitis condensans ilii (Fig 1c) and bone scintigraphy revealed increased radioactive tracer concentration over the painful bony sites (Fig 1d). CT-guided bone biopsy from the right ilium showed osteosclerosis (Fig 1e).

Based on clinical, laboratory, imaging and histopathological findings, the diagnosis of Schnitzler syndrome was undertaken. Interleukin-1-receptor antagonist anakinra 100 mg was administered subcutaneously daily with a remarkable response on fever, bone pain, skin rash and acute phase reactants within 1 week.

Schnitzler syndrome is an acquired autoinflammatory disease sharing similar symptoms with periodic fever syndromes, in 
particular the cryopyrin-associated periodic syndrome. ${ }^{1}$ It presents with fever, urticarial rash (histologically, a neutrophilic dermatosis), bone pain and systemic inflammation, while the presence of a monoclonal IgM is the hallmark. ${ }^{2}$ Diagnosis is based on established criteria. ${ }^{3}$ Ruling-out other diagnoses, like adult-onset Still's disease, Sweet syndrome and hypocomplementemic urticarial vasculitis is essential. It is not known whether the pathological clone results in the activation of the inflammasome or vice versa. AA-amyloidosis is a concern in untreated patients, while $20 \%$ of them might evolve to lymphoproliferative disease. There are controversial reports on patients with Schnitzler syndrome bearing mutation variations of the NLRP3 gene. ${ }^{4}$ This case is presented to alert physicians on its existence since it may mimic systemic autoimmune or autoinflammatory syndromes.

\section{References}

1 Pizzirani C, Falzoni S, Govoni M et al. Dysfunctional inflammasome in Schnitzler's syndrome. Rheumatology (Oxford) 2009;48:1304-8.

2 Vanderschueren S, Van der Veen A. The Schnitzler syndrome: chronic urticaria in disguise: a single-centre report of 11 cases and a critical reappraisal of the literature. Clin Exp Rheumatol 2017;35:69-73.

3 Simon A, Asli B, Braun-Falco M et al. Schnitzler's syndrome: diagnosis, treatment, and follow-up. Allergy 2013;68:562-8.

4 Rowczenio DM, Pathak S, Arostegui JI et al. Molecular genetic investigation, clinical features, and response to treatment in 21 patients with Schnitzler syndrome. Blood 2018;131:974-81.

Address for correspondence: Dr Evangelia Zampeli, 3rd Department of Internal Medicine, Sotiria Hospital, Medical School, University of Athens, 11527 Athens, Greece. Email: zampelieva@gmail.com

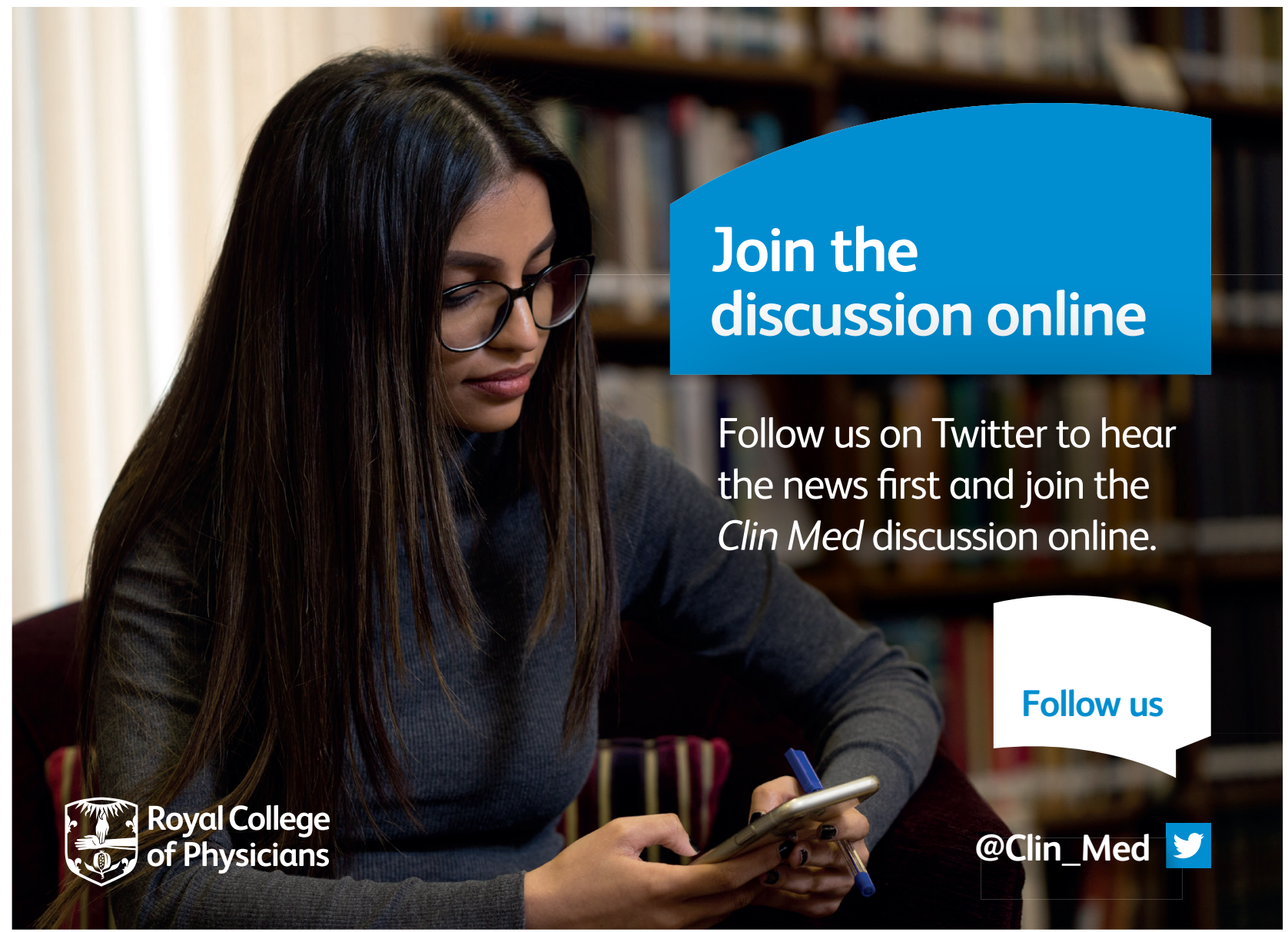

